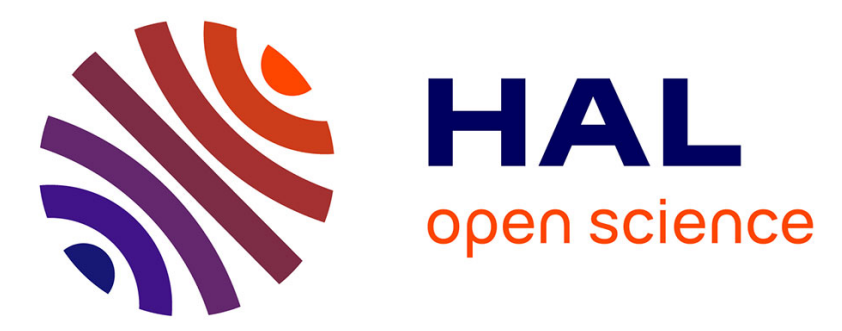

\title{
Elastomer biaxial characterization using bubble inflation technique. I: Experimental investigations
}

\author{
N Reuge, Fabrice Schmidt, Yannick Le Maoult, M Rachik, F Abbe
}

\section{To cite this version:}

N Reuge, Fabrice Schmidt, Yannick Le Maoult, M Rachik, F Abbe. Elastomer biaxial characterization using bubble inflation technique. I: Experimental investigations. Polymer Engineering and Science, 2001, 41 (3), pp.522-531. 10.1002/pen.10749 . hal-01709517

\section{HAL Id: hal-01709517 \\ https://hal.science/hal-01709517}

Submitted on 19 Mar 2019

HAL is a multi-disciplinary open access archive for the deposit and dissemination of scientific research documents, whether they are published or not. The documents may come from teaching and research institutions in France or abroad, or from public or private research centers.
L'archive ouverte pluridisciplinaire HAL, est destinée au dépôt et à la diffusion de documents scientifiques de niveau recherche, publiés ou non, émanant des établissements d'enseignement et de recherche français ou étrangers, des laboratoires publics ou privés. 


\title{
Elastomer Biaxial Characterization Using Bubble Inflation Technique. I: Experimental Investigations
}

\author{
N. REUGE ${ }^{1}$, F. M. SCHMIDT ${ }^{2, *}$, Y. LE MAOULT ${ }^{2}$, \\ M. RACHIK ${ }^{3}$, and F. ABBE ${ }^{4}$ \\ ${ }^{1}$ Laboratoire des composites thermostructurawx \\ Domaine universitaire 3, Allée de la Boétie \\ 33600 Pessac, France \\ ${ }^{2}$ Ecole des Mines d'Albi-Carmaux \\ Campus Jarlard, Route de Teillet \\ 81013 Albi CT Cedex 09, France \\ ${ }^{3}$ Université Technologique de Compiègne \\ BP649 60206 Compiègne Cedex, France \\ ${ }^{4}$ SNECMA, Division SEP \\ Le Haillan BP 3733135 Saint-Médard en Jalles, France
}

\begin{abstract}
The biaxial rheological behavior of materials such as elastomers or polymers can be obtained using a bubble-inflation-technique. A circular membrane is clamped at the rim and expanded under gas pressure. The inflation of the circular membrane is recorded using a $C C D$ video camera and the blowing pressure by a pressure sensor. Then, from elongation and curvature radius measurements at the pole of the bubble, one can deduce equibiaxial stress-strain data. This study describes the optimization of a bubble-inflation rheometer. The most sensitive point of the technique is the estimation of the elongation at the bubble pole, deduced from video camera measurements. A direct measurement of the bubble thickness was performed using a magnetic probe in order to validate rheometer results. Such a validation has evidently never been carried out before. Results of quasi-static equibiaxial characterization of elastomers are presented and analyzed.
\end{abstract}

\section{1) INTRODUCTION}

$\mathrm{T}$ here is a growing interest in determining biaxial properties of materials such as elastomers used for mechanical applications or thermoplastics for the simulation of plastic-processing operations such as blow molding, stretch blow molding or thermoforming (1). In these specific processes, the polymer is heated above the glass-transition temperature but far from the melting point. Consequently, the biaxial rheometer should be suitable for a material close to a solidlike state. In addition, the characterization of elastomers is a difficult task because of their high deformation ability and their complex behaviors (nonlinear elasticity, viscoelasticity, crystallization, instability, and so on).

Different types of biaxial rheometers are used toward this end. The classic technique of biaxial plane extension of cruciform samples is not really suitable for such materials. The Meissner rheometer (2), made of rotary clamps, seems to be more suitable (but complex and expensive, especially in multiaxial configuration). Another way to characterize such materials is the squeeze-flow-technique, where a sheet of sample is compressed. A special lubricant is required between the sample surfaces and the compressing plates in order to avoid any shear flow within the sample. But this rheometer is more convenient for liquid-like materials. 
Ultimately, bubble inflation seems to be the best way to carry out an equibiaxial elongation of elastomers with a relatively simple device.

The inflation of circular membranes has been of interest to scientists and engineers for many years. Bubble inflation with compressed air was used for the first time by Treolar (3) in 1944, to study the behavior of natural rubber and its mechanisms of bursting. In 1951, Rivlin et al. (4) used this technique to identify Neo-Hookean and Mooney-Rivlin rheological parameters, and showed that the equibiaxial state of deformation at the pole bubble is equivalent to compression according to the normal axis of the membrane. In 1971, Alexander (5) studied and modeled atmospheric neoprene balloon instabilities using the bubble inflation technique. In 1973, Paisant (6) studied the deformation of natural rubber and SBR membranes to model their biaxial behaviors.

Polymer materials have been also investigated with this technique. In 1975, Schmidt and Carley (7) developed numerical models using non-linear constitutive equations issued from the rubber-like materials theory for the membrane-inflation problem and compared their results with experimental values obtained with high-impact polystyrene and cellulose acetate butyrate above their respective glass-transition temperatures. In 1980, Denson and Hylton (8) used oil to inflate ABS membranes near the forming temperature $\left(138^{\circ} \mathrm{C}\right)$. Oil being incompressible, they could carry out retardation experiments, which were impossible with air. In 1992, Thomas and Tintillier (9) used a video camera system (CCD camera) and preprinted membranes to analyze equibiaxial deformations of PET membranes heated above the glass transition temperature. In 1994, Kappel (10) used the same kind of device to study an elastomer. They reached promising results, but the equibiaxial rheometer is not yet really operational.

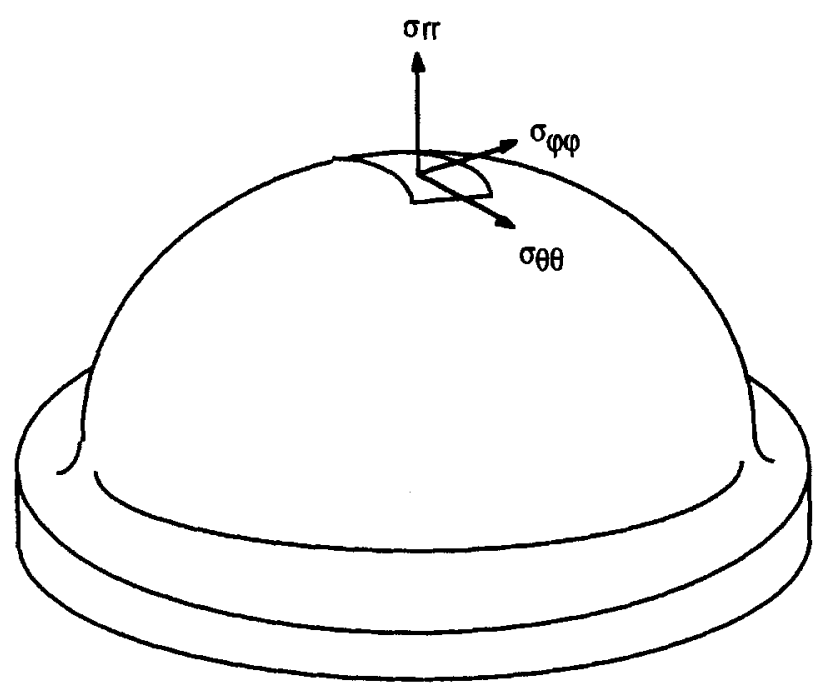

Fig. 1. Inflated bubble in biaxial extension.
In 1996, Michaeli et al. (11) developed a specific bubble inflation rheometer, in which again, a membrane is inflated using silicon oil with some improvements to prescribe a constant strain rate during the entire process.

In 1997, Verron (12) developed a numerical procedure using bubble height to compute the rheological parameters for a given constitutive equation during membrane inflation. This numerical approach does not require a complex video camera device to mark the membrane, but the method requires the knowledge of the constitutive equation.

Our goal is to develop and optimize a rheometer based on a bubble-inflation technique similar to those of Thomas, Tintillier and Kappel. For this, we have proceeded to comparisons with the measured thickness of the inflated membrane using the inflation device and a magnetic probe thickness measurement. The inflation rheometer was then applied to determine the biaxial rheological properties of elastomers at ambient temperature (no heating stage).

\section{2) BUBBLE INLATION ASSUMIPTONS}

\section{1) Hoop Stress Calculation}

A uniform circular membrane is clamped at the rim and inflated using compressed air applied to one side in order to produce a bubble as sketched in Fig. 1 . The inflation of the circular membrane results in a truly biaxial stretching at the pole of the bubble and a planar extension near the rim. The assumption of spherical symmetry allows us to equate $\sigma_{\theta \theta}$ to $\sigma_{\varphi \varphi}$ at the pole of the bubble. So, the Cauchy stress tensor o expressed in spherical coordinates reduces to:

$$
\underline{\underline{\sigma}}=\left[\begin{array}{ccc}
\sigma_{\pi} & 0 & 0 \\
0 & \sigma_{\theta \theta} & 0 \\
0 & 0 & \sigma_{\theta \theta}
\end{array}\right]_{(r, \theta, z)}
$$

The ratio between the thickness of the inflated membrane $e$ and the curvature radius $R_{c}$ being small enough $(13,14)$, the thin shell assumption allows us to neglect the radial stress $\sigma_{r}$ in front of the hoop stress $\sigma_{\theta \theta}$. In addition, we equate $\sigma_{\theta \theta}$ to the thicknessaverage hoop stress, which leads to:

$$
\sigma_{\theta \theta}=\frac{\Delta P R_{c}}{2 e}+\sigma_{o}
$$

assuming that the thickness is uniform at the pole of the bubble. $\Delta P$ is the differential inflation pressure. $\sigma_{0}$ is an additional stress due to the initial compression of the membrane.

\section{2) Thidoness Calculation}

The differential inflation pressure may be easily recorded using a pressure sensor and the curvature radius measured using video camera images analysis in order to calculate the hoop stress ( $E q$ 2). For the thickness calculation, we need to locate a marked 


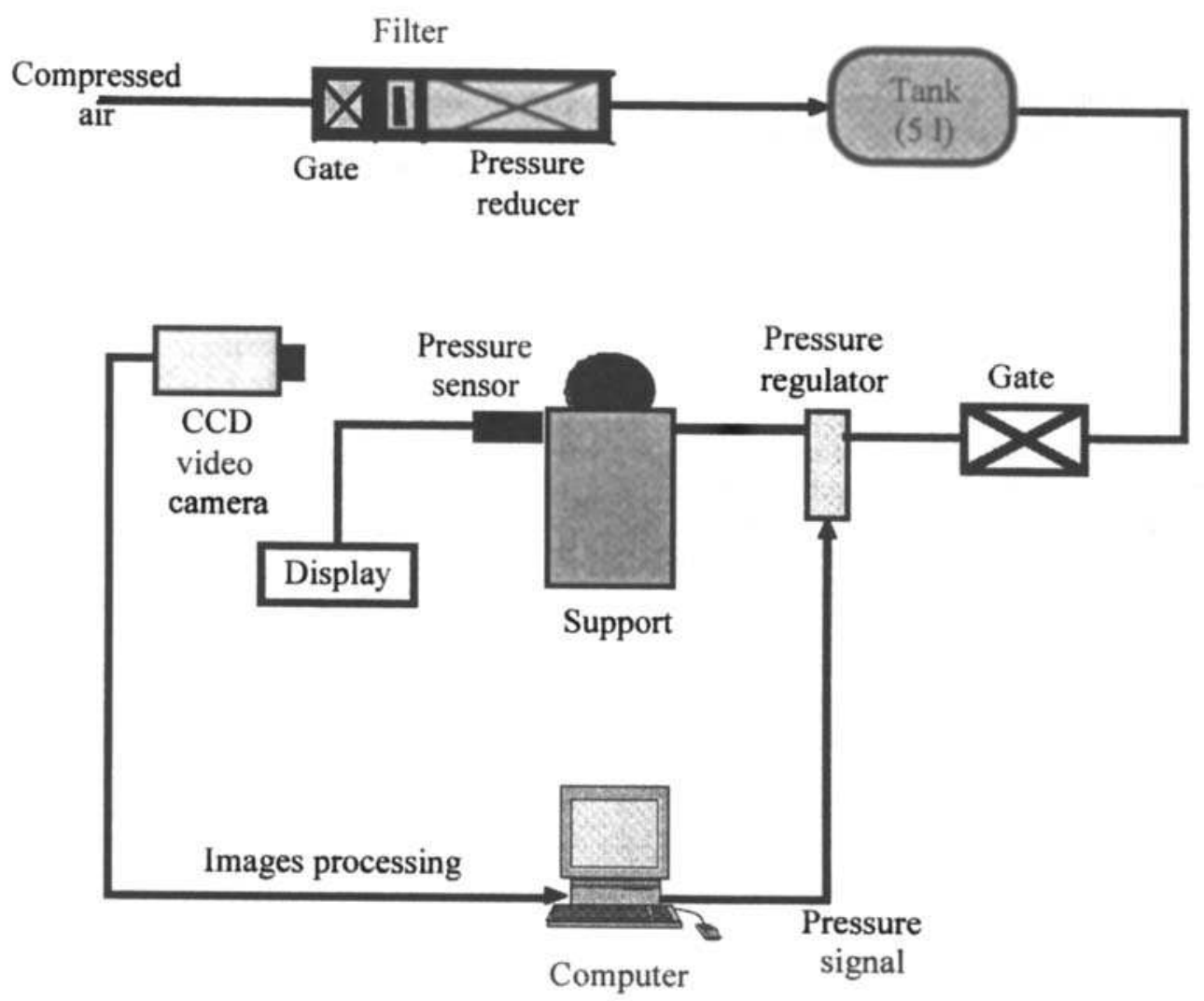

Fig. 2. Schematic view of the bubble inflation rheometer.

area around the pole of the bubble where the deformation of the membrane is equibiaxial and the thickness uniform (See Section 3).

Using the volume balance at a local level, the thickness may be computed using the area change:

$$
\boldsymbol{e}=\frac{e_{0}}{\lambda_{\theta \theta}^{2}}
$$

where $e_{0}$ is the flat circular membrane thickness. The hoop stretch $\lambda_{\theta \theta}$ is the ratio between the current length of the profile contour of the polar zone $l$ and the initial length of the polar zone $b_{0}$ :

$$
\lambda_{\theta \theta}=\frac{l}{l_{0}}
$$

Then, the thickness-average hoop stress leads to:

$$
\sigma_{\theta \theta}=\frac{\Delta P R_{c} \lambda_{\theta \theta}^{2}}{2 e_{0}}+\sigma_{0}
$$

\section{3) BUBBLE INFLATION TECENIgUE}

A schematic view of the bubble inflation rheometer is presented in Fig. 2. A circular membrane is mounted between two metal disks containing a circular aperture (40 mm diameter) and clamped on a support. The membrane has a $60-\mathrm{mm}$ diameter and a $2-\mathrm{mm}$ thickness. The air pressure is regulated with a very accurate device and recorded using a pressure sensor located close to the backside of the membrane. The inflation of the membrane is recorded using a $C C D$ SONY video camera $(752 \times 582$ pixels). A PC computer is used to send a signal to the pressure regulator and to acquire images of video camera by a MATROX image grabber (24 frames/s). Elastomeric membranes were successfully inflated beyond the hemispherical stage to large elongation up to 4 (Fig. 3). It appears immediately that the hemispherical deformation occurs only at low elongation $(\leq 2.5)$

A very sensitive point is that elongation and curvature radius must not be measured on the entire bubble contour because deformation of the membrane is rigorously equibiaxial only at the pole of the bubble. Consequently, we had to mark the bubble in order to locate a polar area where equibiaxiality can be considered (this assumption will be verified in Section 4). A viscous silicon paste was used to perform a rectangular white mark with an initial width of $10 \mathrm{~mm}$. So, the polar area could be extracted, the length of its contour measured, and its curvature radius computed using a least squares fit (Fig. 4). These operations were carried out by our image-processing software (15). Finally, the hoop stretch and stress could be deduced from videometric and pressure measurements. 


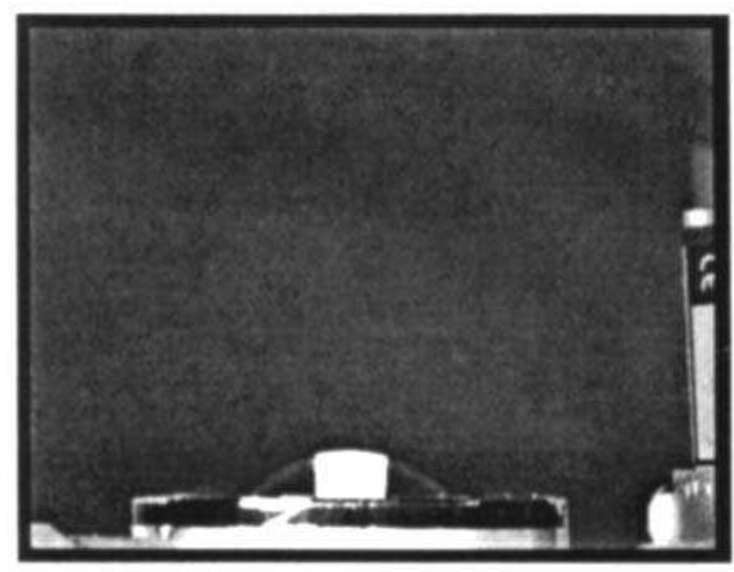

$\mathrm{P}=0.288$ bar $\left(\lambda_{00}=1.17\right)$

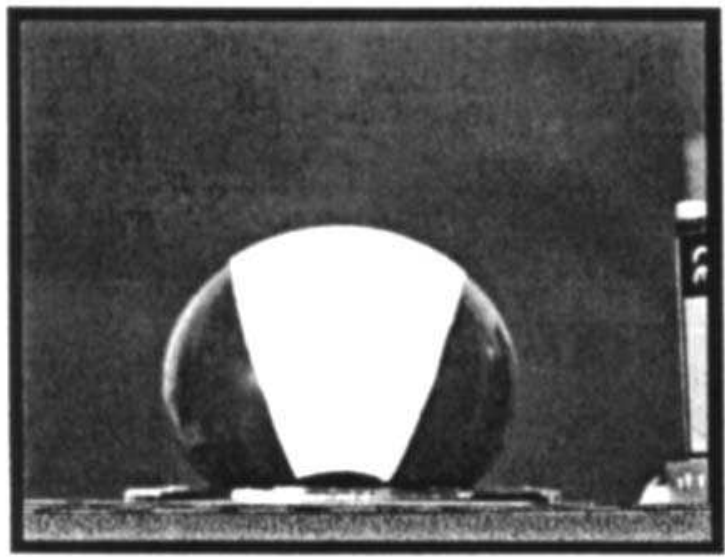

$P=0.605$ bar $\left(\lambda_{\theta \theta}=4.25\right)$

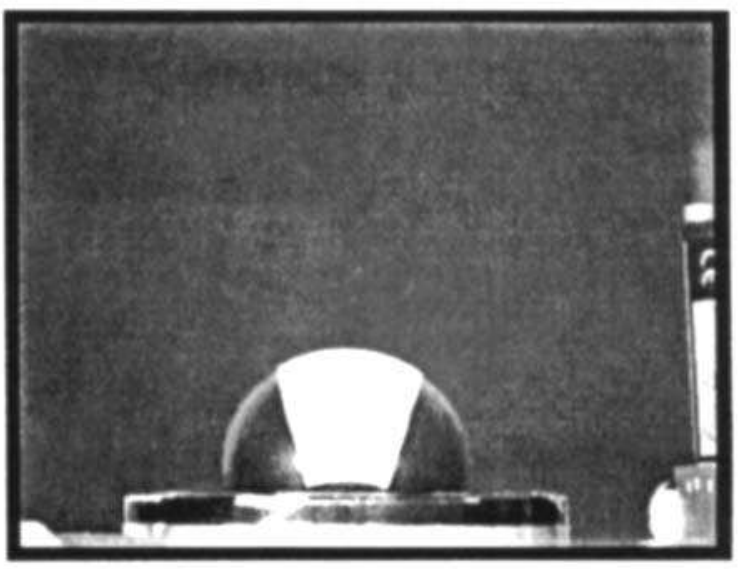

$\mathrm{P}=0.598 \mathrm{bar}\left(\lambda_{00}=2.52\right)$

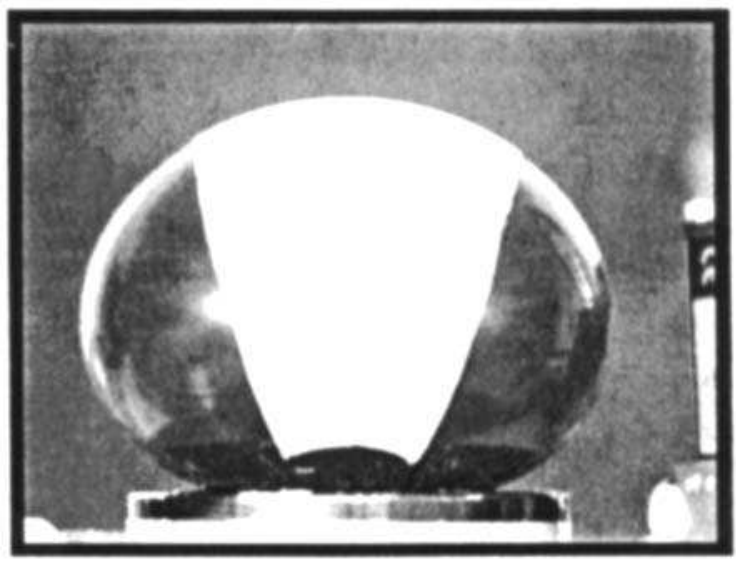

$P=0.799$ bar $\left(\lambda_{\theta \theta}=5.85\right)$

Fig. 3. Intermediate bubble shapes.

\section{4) BIAXTAX RETOMTTER ASSESSWENT}

An experimental setup was carried out in order to validate the polar area assumption. For this, we have to compare the thickness measured at the pole of the bubble using the bubble-inflation rheometer (video camera measurement) and the thickness measured directly by another device, using a magnetic probe. Such a validation seems never to have been carried out before.

The standard technique used for the thickness measurement requires a magnet ball and a magnetic probe (16). The magnet ball is placed upon the membrane and the clamping system is reversed (Fig. 5). The magnetic field measured by the probe depends only on the distance between the probe and the ball, which represents the thickness of the membrane. For a $2-\mathrm{mm}$ thick sample, the uncertainty of this device is $\pm 30 \mu \mathrm{m}$. Then, the membrane is inflated for increasing pressure values. For each pressure value, the magnetic probe fixed on a rack is in perfect contact with the pole of the reversed bubble. We proceed to five measurements for each pressure value. The pole bubble height $H$ is measured using the video camera image analysis.

\section{1) Sphorical Bubble-Inflation Assumption}

If we assume that the bubble remains spherical during inflation (the curvature radius and the thickness are assumed to be constant), the volume balance on the entire bubble (asoumption 1) permits calculation of the relative thickness $\frac{e}{e_{0}}$ :

$$
\frac{e}{e_{0}}=\frac{R_{0}^{2}}{R_{0}^{2}+H^{2}}
$$

where $R_{0}$ is the useful membrane radius. Now, using the volume balance at the pole of the bubble (assumption 2) yields: 

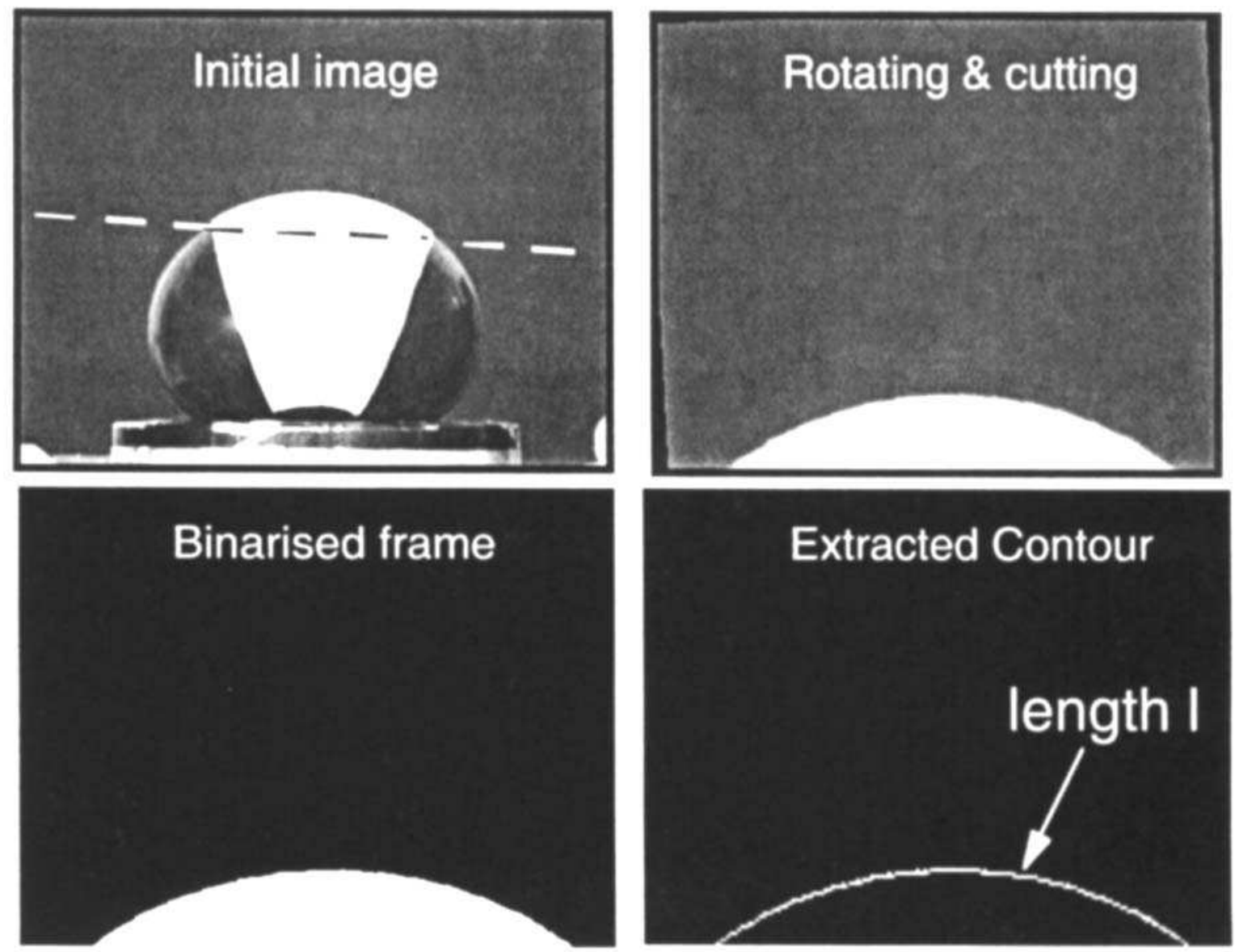

Fig. 4. Video camera images processing.

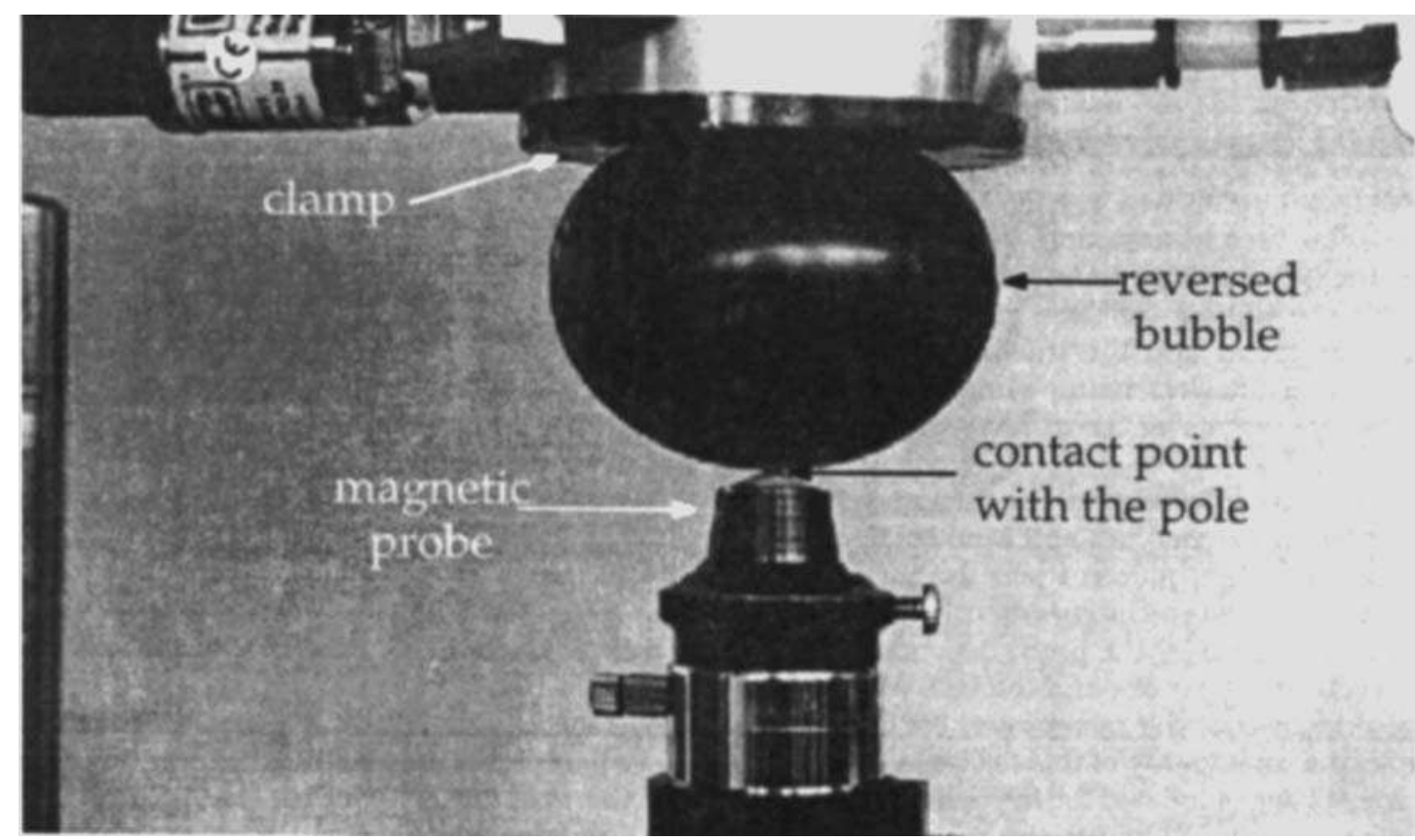

Fig. 5. Magnetic probe in contact with the pole. 


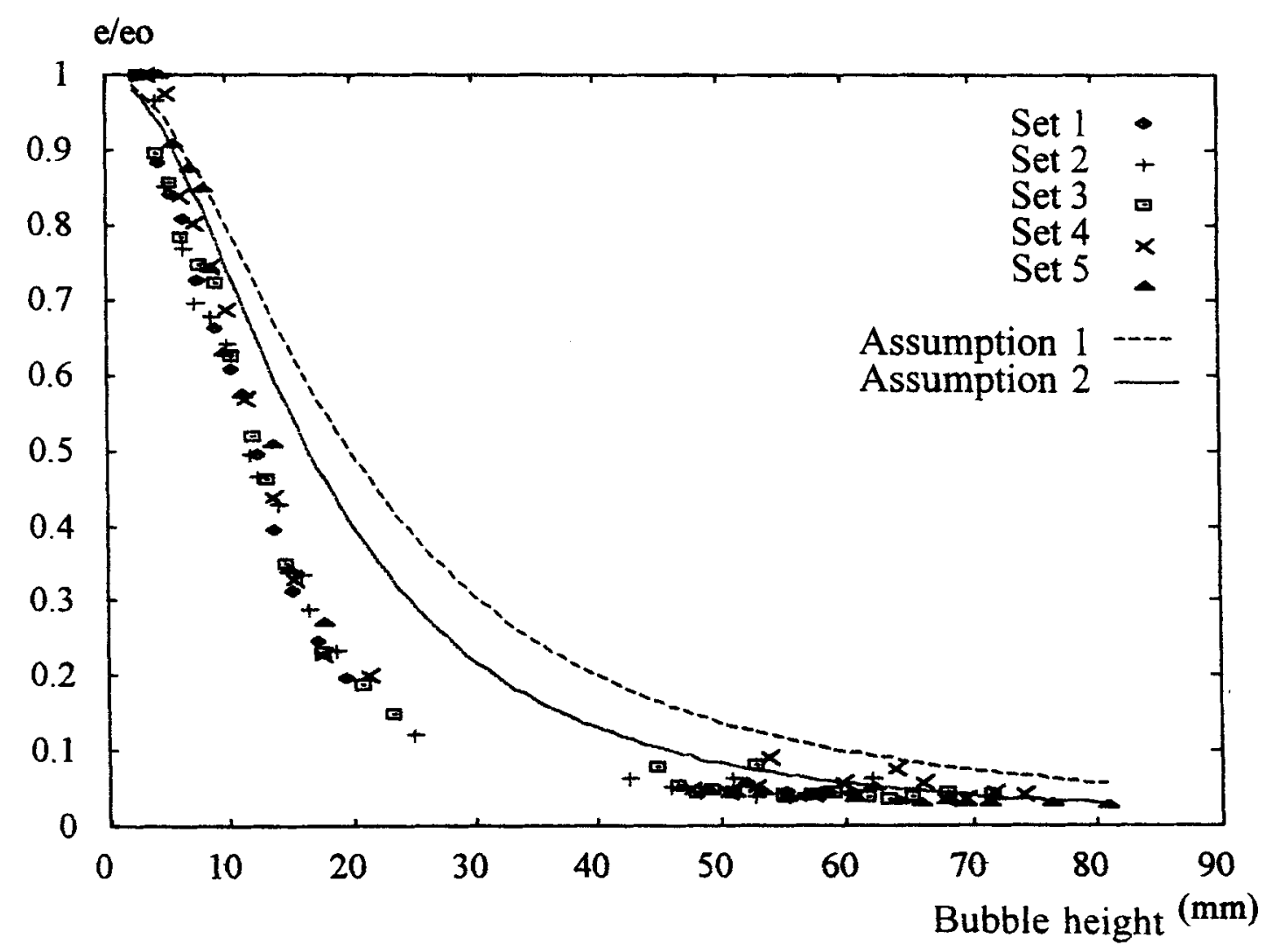

Fig. 6. Comparison between measured and calculated relative thickness versus bubble height.

$$
\frac{e}{e_{0}}=\frac{R_{0}^{2}}{\left(\frac{\mathrm{R}_{0}^{2}+H^{2}}{2 H}\right)^{2} \operatorname{arc\operatorname {cos}^{2}(1-\frac {2\mathrm {H}^{2}}{\mathrm {R}_{0}^{2}+\mathrm {H}^{2}})}}
$$

In Fig. 6, the relative thickness $\frac{e}{e_{0}}$ measured using the bubble-inflation rheometer and using assumptions 1 and 2 are plotted versus bubble height. In both cases, the error between the measured thickness and the calculated thickness is important. The maximum error $\frac{\Delta e}{e}$ is more than $100 \%$. Thus, the spherical bubble assumption together with the volume balance assumption will not yield useful values for this application. Note that for the assumption 2, the discrepancy is lower than for the assumption 1.

\section{2) Polar Area Assumption}

In Fig. 7, the relative thickness is plotted versus bubble height for the magnetic probe thickness and the rheometer thickness. Three different elongation domains appear:

- a domain where the elongation is low/medium $\left(\lambda_{\theta \theta}\right.$ $\leq 2.5$ i.e. $\mathrm{H} \leq 28 \mathrm{~mm}$ ) and for which the discrepancy between the two methods is less than 10\%; the agreement is fair and allows validation of the rheometer measurement;
- an instability zone where no measurement is possible; this phenomenon is related to the stress-induced crystallization of the elastomer during the biaxial elongation and will be analyzed in section $\mathbf{5}$;

- in the last domain of large elongation $\left(\lambda_{\theta \theta} \geq 4.25\right.$ i.e. $H \geq 42 \mathrm{~mm}$ ), the uncertainty of the magnetic probe measurement increases as a result of local variations of the thickmess (the sample slides slightly between the clamps).

\section{5) APPLICATIONS}

\section{1) Equibiadal Theological Beharior of an NR/SBR Elantomer}

We present the equibiaxial stress/strain data for a natural rubber/SBR compound, used by SNECMA-SEP for mechanical applications (flexible pieces) obtained for static inflation at ambient temperature $\left(25^{\circ} \mathrm{C}\right)$. This elastomer is a natural rubber (NR) sulfur cured (SEV system) filled with carbon black. For reasons of confidentiality, the exact composition of this material cannot be given. The pressure is imposed at a given value. The different parameters are calculated using the image processing software associated to the bubble inflation rheometer (inflated membrane thickness, curvature radius, hoop stress and stretch). The procedure is repeated until the elastomer limit of elongation 


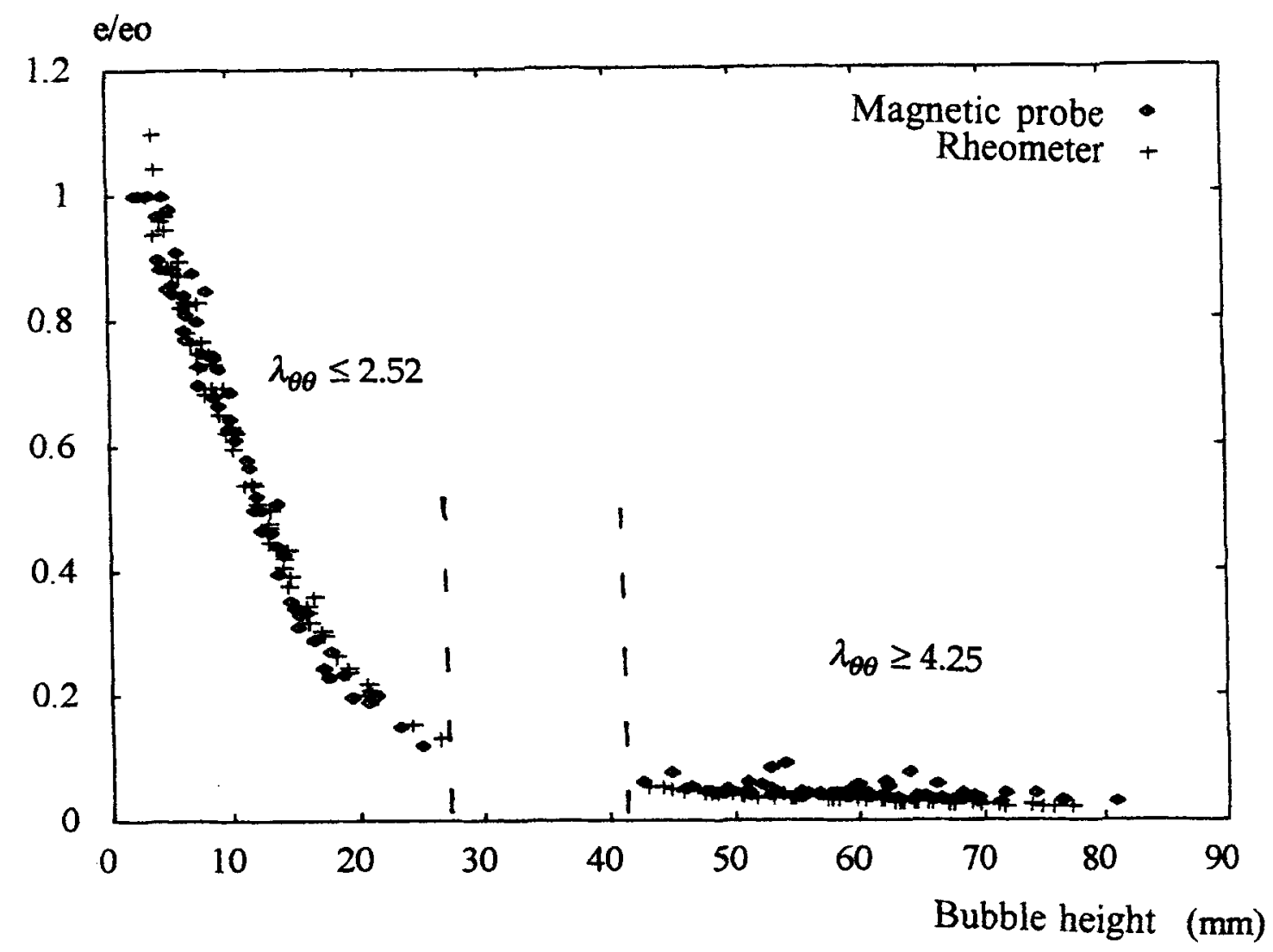

Fig. 7. Comparison between magnetic probe and rheometer relative thickness versus bubble height.

is reached (before bursting). We proceed to five measurements for each pressure value. Note that the uncertainty for the measurements is less than $4 \%$, which is quite fair.

In Fig. 8, equibiaxial stress-elongation data of the studied elastomer are plotted. Again, three different elongation domains appears:

- a domain where the elongation is low/medium $\left(\lambda_{\theta \theta}\right.$ $\leq 2.5$ ) and for which the rheological behavior of the elastomer is quasi-linear;

- an instability zone where no measurement is possible;

- a last domain of large elongation $\left(\lambda_{\theta \theta} \geq 4.25\right)$, where strain hardening occurs.

At small and medium deformations, chain segments of the NR-network are linearly extended. The stressstrain curve is linear. Then, the domain of instability begins and the bubble inflates quickly at constant pressure. Figure 3 illustrates this phenomenon: image $\mathbf{3 b}$ shows the bubble just before the instability and image $3 c$ the bubble just after the instability; the elongation goes from 2.5 to 4.25 as the pressure remain constant at 0.6 bar. This phenomenon is well known for inflation of NR membrane and can be attributed to strain-induced crystallization (see Section 5.2).
In Fig. 9, the mean curve for equibiaxial stress-elongation of the elastomer studied is plotted and compared with the uniaxial stress-elongation curve. The tensile test was performed with an Instron 1100 equipment using a standard H2 sample (NFT 46-002). The elongation speed was fixed to $1 \mathrm{~mm} / \mathrm{min}$. In that case, uniaxial and equibiaxial curves are very similar (the maximum discrepancy is $15 \%$ ). This is not the case if we plot the true stress-strain data (Fig. 10). Depending on the type of elongation. the true strain and stress are defined below:

- Uniaxial elongation

$$
\bar{\varepsilon}=\varepsilon_{z z}, \bar{\sigma}=\sigma_{z z}
$$

- Equibiaxial elongation

$$
\bar{\varepsilon}=2 \varepsilon_{\theta \theta}, \bar{\sigma}=\sigma_{\theta \theta}
$$

We have now for a given value of true stress the equibiaxial true strain that is two times the uniaxial true strain.

\section{2) Stress-Induced Crystallization During Elongation}

Mullins et al. $(17,18)$ have reported on an instability phenomenon for natural rubber during tensile 


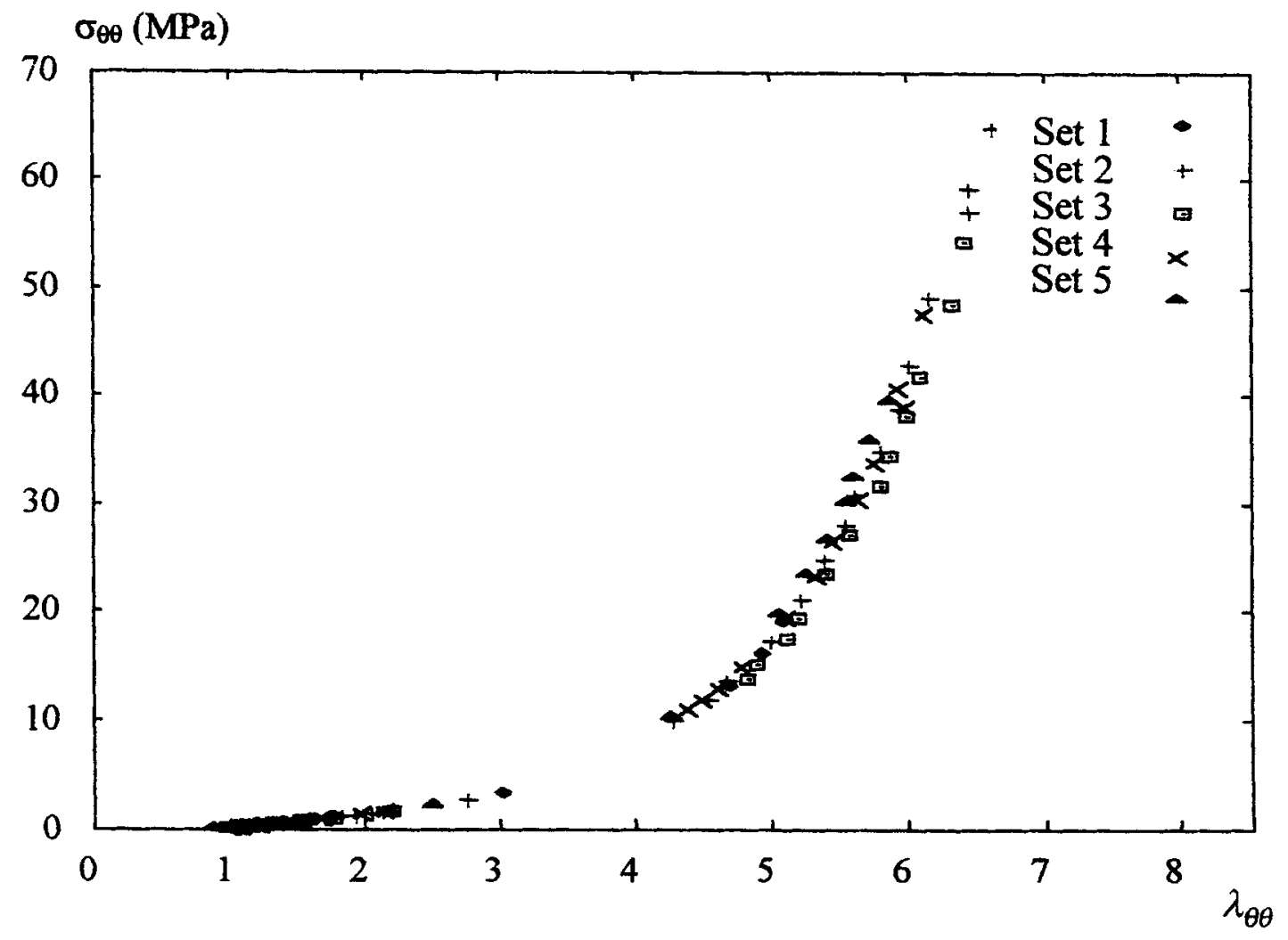

Fig. 8. Hoop equibiaxial stress-elongation data $\left(T=25^{\circ} \mathrm{C}\right)$.

$\sigma_{\theta \theta}, \sigma_{\mathrm{zz}}(\mathrm{MPa})$

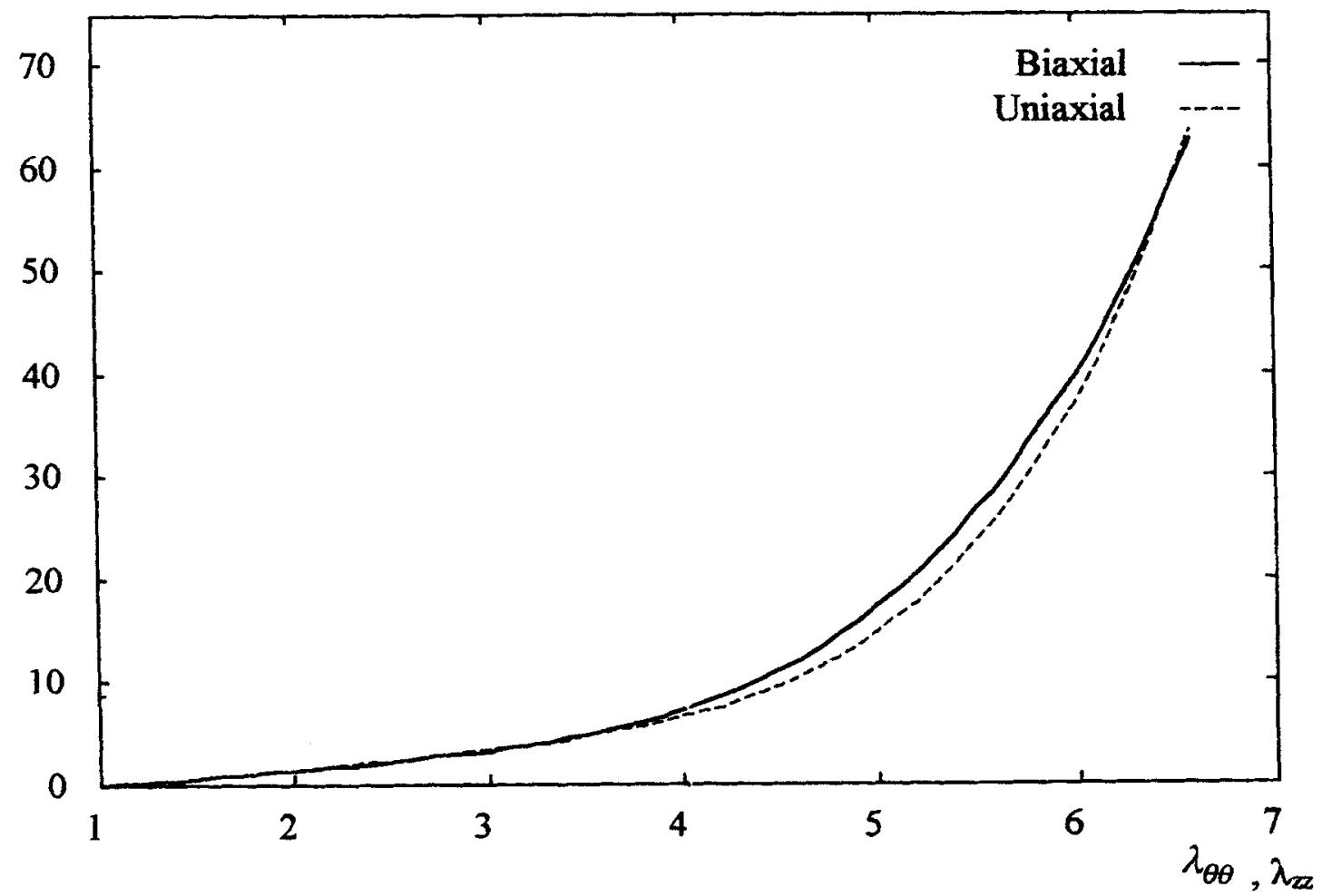

Fig. 9. Comparison between biaxial and uniaxial stress-elongation data for a natural rubber, $\mathrm{SBR}$ compound $\left(T=25^{\circ} \mathrm{C}\right)$. 


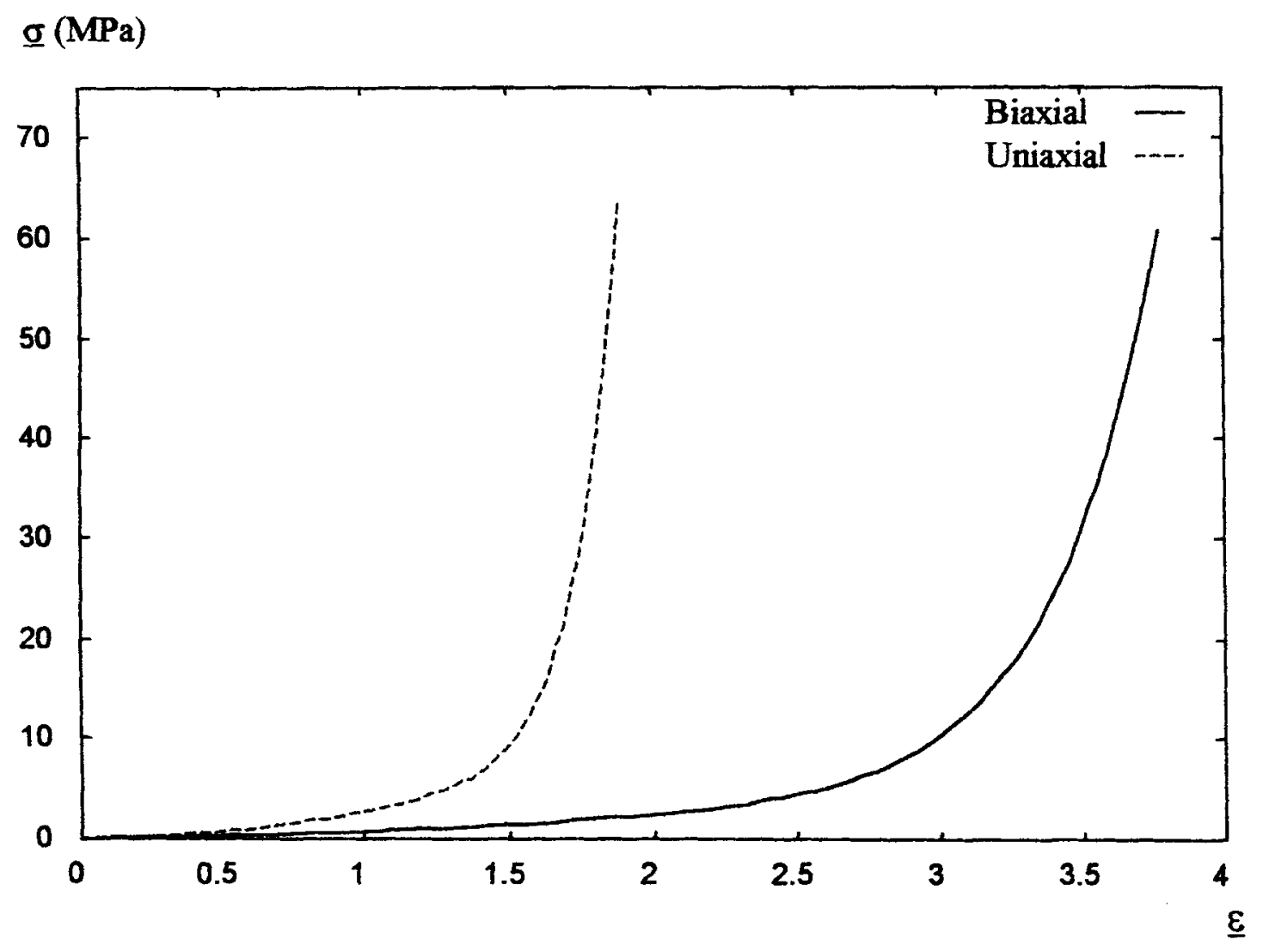

Fig. 10. Comparison between biaxial and uniaxial true stress-strain data for a natural rubber, $\mathrm{SBR}$ compound $\left(T=25^{\circ} \mathrm{C}\right)$.

tests and balloon inflation similar to the phenomenon we have observed. They point out that the instability zone is related to the stress-induced crystallization during elongation at ambient temperature. In addition, they observe that crystallization appears first where stresses and deformations are maximum (the center of the tensile sample and the pole of the balloon). Then the crystallization front is progressively propagated in the whole natural rubber. In particular, they point out stress softening for crystallizing elastomers when the same sample is submitted to successive elongation cycles. This effect is the so-called Mullins effect. We have submitted the natural rubber/ SBR compound elastomer to three successive elongation cycles, and we observe the same behavior as plotted in Fig. 11. This simple experience demonstrates that the instability phenomenon for this elastomer is due to crystallization during elongation.

Another effect induced by the crystallization is strain hardening. Because of the stronger alignment of chain segments, crystallization appears preferably at locations of large strain or stress, respectively. and leads to a reinforcement of these weakest domains of the network. The resulting improved tensile strength properties are typical for NR-networks and a consequence of the high cis-portion in this rubber, related to the ability to crystallize, together with the very slow crystal growth velocity $(19,20)$.

\section{6) CONCLusion}

A bubble-inflation rheometer has been developed and optimized. We have validated the thickness computed using the area change by measuring the thickness using a standard magnetic probe. In addition, we demonstrate that the spherical bubble assumption together with the volume balance assumption will not yield useful values for this application.

Then, biaxial rheological characterization of a natural rubber/SBR compound elastomer was conducted using this rheometer. For this elastomer, the biaxial behavior is very similar to the uniaxial behavior. It should be noted that the comparison depends upon whether we use the true stress and strain or the uniaxial and hoop stress and strain. The stress-induced crystallization phenomenon has been highlighted.

Future works will allow us to proceed to dynamic inflation by using a high-speed video carnera and the relevant image grabber. In order to proceed to polymer inflation, an air convection oven will be added to the present bubble-inflation rheometer. This will allow us 


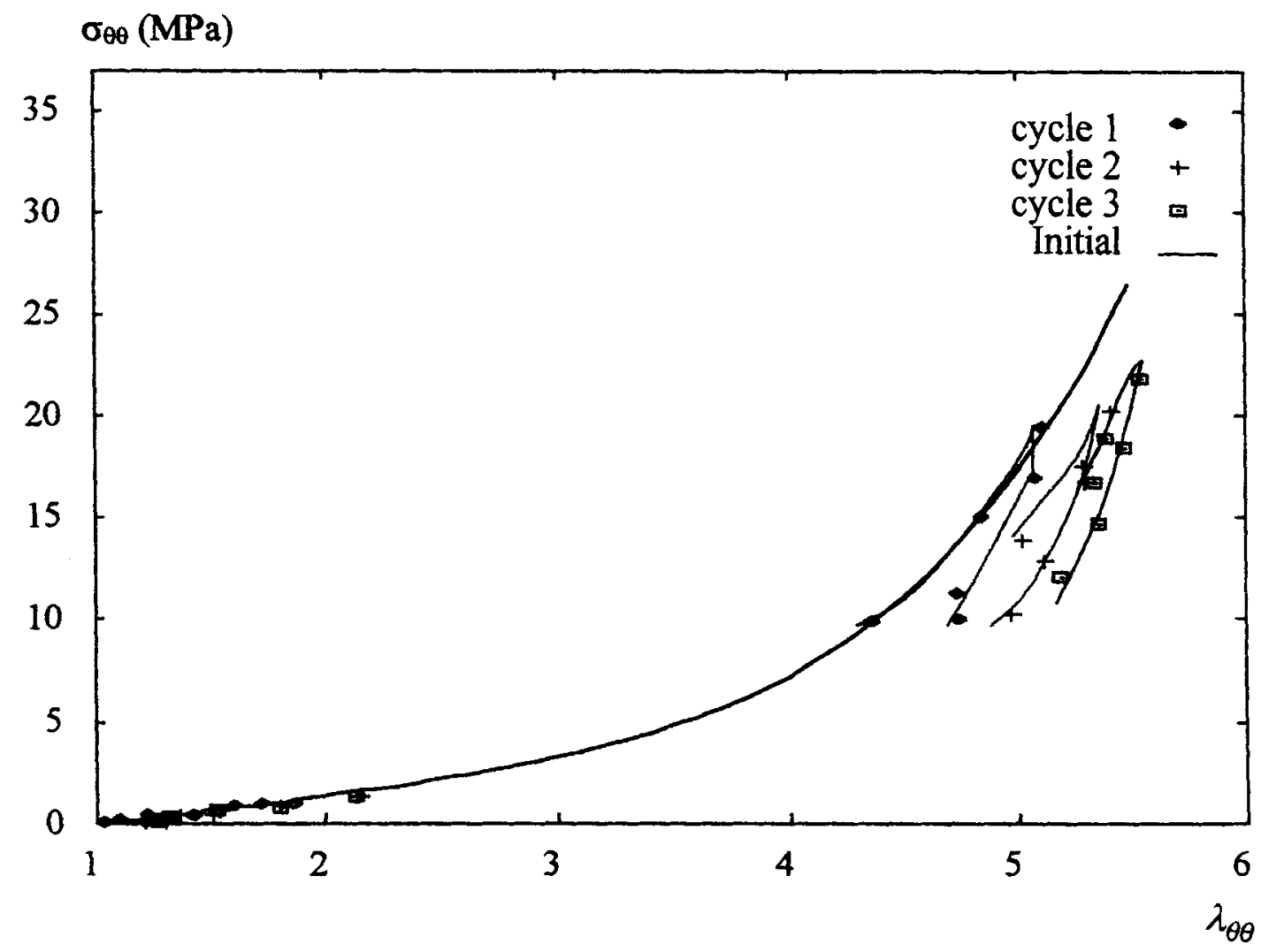

Fig. 11. Stress-softening for the elastomer after successive elongation cycles.

to heat polymers above the glass transition temperature and to obtain biaxial stress-strain data for polymers.

\section{ACKTOWLEDGMENT}

SEP-Division SNECMA Company supported this research. Special thanks go J. P. Arcens, T. Lopez and B. David.

\section{RETERENCES}

1. H. G. Delorenzi and H. F. Nied, Computers \& Structures, 28, 197 (1987).

2. J. Meissner, Polym. Eng. Sci, 27 (1987).

3. L. R. G. Treolar, Trans. Inst. Rubber Ind, 18, 201 (1944).

4. R. S. Rivlin and D. W. Saunders, Phil. Trans. R. Soc., A243, 251 (1951).

5. H. Alexander, Int. J. Engng. Sci, 8, 151 (1971).

6. J. C. Paisant, "Aspect rhêologique du gonflement d'une membrane en caoutchouc, mesure des configurations de forme" (in French), Industrie Minérale, 115 (1973).

7. L. R. Schmidt and J. F. Carley, Polym. Eng. Sci. 15, 51 (1975).

8. C. D. Denson and D. C. Hylton, Polym. Eng. Sci, 20, 535 (1980).
9. L. Thomas and M. Tintillier, "Développement et mise au point d'une méthode d'essai de bullage de flans en $P . E$. T avec mesure vidéométrique" (in French), DEA Report, ENSTIMD (1992).

10. H. Kappel, "Développement d'une méthode expérimentale d'étude du comportement des matériaux en déformation biaxiale symétrique" (in French), Report, ESSTIN (1994).

11. W. Michaeli and K. Hartwig, Kunststoffe, 86 (1996).

12. E. Verron, "Contribution expérimentale et numérique au procédés de moulage par soufflage et thermoformage" (in French), Thesis, ECN (1997).

13. F. M. Schmidt, J. F. Agassant, M. Bellet, and L. Desoutter, J. Non-Newt. Fluid Mech, 64, 19 (1996).

14. F. M. Schmidt, J. F. Agassant, and M. Bellet, Polym. Eng. Sci., 38, 1399 (1998).

15. N. Reuge, "Mise au point et optimisation d'un rhéomètre équibiaxial pour la caractérisation d'un élastomère" (in French), Report, EMAC (1998).

16. G. Lejeune, "Contrôle d'épaisseur" (in French), Technique de l'ingénieur, R-1370, 16, (1999).

17. L. Mullins, J. Rubber Research, 16, 275 (1947).

18. L. Mullins, J. A. C. Harwood, and A. R. Payne, J. IRI, 17 (1967).

19. M. Kluppel, Kautschuk und Gummi, Kunststoffe, 47, 242 (1994).

20. L. Mandelkern, Rubber Chemistry and Technology, 68 , 61 (1993). 